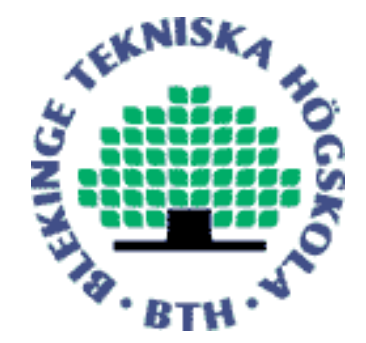

Copyright (C) 2014 IEEE.

Citation for the published paper:

Performance Analysis of Randomized Distributed Space-Time Codes over Composite Gamma/Lognormal Fading Channels

Jacopo Soffritti, Trung Q. Duong, Maria Luisa Merani, Hans-Jürgen Zepernick

IEEE Vehicular Technology Conference

2014 Vancouver, Canada

This material is posted here with permission of the IEEE. Such permission of the IEEE does not in any way imply IEEE endorsement of any of BTH's products or services Internal or personal use of this material is permitted. However, permission to reprint/republish this material for advertising or promotional purposes or for creating new collective works for resale or redistribution must be obtained from the IEEE by sending a blank email message to pubs-permissions@iee.org.

By choosing to view this document, you agree to all provisions of the copyright laws protecting it. 


\title{
Performance Analysis of Randomized Distributed Space-Time Codes over Composite Gamma/Lognormal Fading Channels
}

\author{
Jacopo Soffritti ${ }^{\dagger}$, Trung Q. Duong ${ }^{\ddagger}$, Maria Luisa Merani ${ }^{\star}$ and Hans-Jürgen Zepernick ${ }^{\dagger}$ \\ ${ }^{\dagger}$ Blekinge Institute of Technology, Sweden \\ ${ }^{\ddagger}$ Queen’s University Belfast, United Kingdom \\ ${ }^{\star}$ Università degli Studi di Modena e Reggio Emilia, Italy \\ e-mail: jacopo.soffritti@gmail.com, marialuisa.merani@unimore.it, hjz@bth.se, trung.q.duong@qub.ac.uk
}

\begin{abstract}
This work investigates the end-to-end performance of randomized distributed space-time codes with complex Gaussian distribution, when employed in a wireless relay network. The relaying nodes are assumed to adopt a decode-and-forward strategy and transmissions are affected by small and large scale fading phenomena. Extremely tight, analytical approximations of the end-to-end symbol error probability and of the end-toend outage probability are derived and successfully validated through Monte-Carlo simulation. For the high signal-to-noise ratio regime, a simple, closed-form expression for the symbol error probability is further provided.
\end{abstract}

\section{INTRODUCTION}

Cooperative communications and relay networks are gaining greater attention and interest in modern wireless communication systems. These solutions are particularly appealing whenever power and spectrum resources are limited or transmission is subject to severe impairments caused by deep fades and shadowing: in addition to the source and the destination, these schemes leverage the transmissions of further nodes that actively contribute to the data delivery process, therefore improving overall performance. This paper focuses on a multirelay cooperative architecture for point-to-point communications, assuming that the relays operate in half-duplex mode and that they adopt a decode-and-forward protocol. It also makes the assumption that no channel state information (CSI) is available and that there is no communication link among relays.

The literature offers several contributions in the area of cooperative communications via multiple relays: to name a few, the work in [1] applies a linear dispersion space-time code among the relays, a viable approach for a fixed number of relays; the proposal in [2] puts forth a solution named random distributed space-time codes (RDSTC), where each relay transmits a random linear combination of the columns of a deterministic space-time codeword, an appealing alternative for the real setting, where the number of cooperating nodes is a priori unknown. Among the most recent works, it is worth mentioning the study in [3], that investigates the physical layer of an amplify-and-forward relaying protocol whose most notable feature is the ease of implementation; [4], that establishes a cross-layer design also exploiting the medium access control layer benefits of cooperative relaying; [5], that analyzes the performance of optimum combining in a decode-and-forward relay network with equal-power interferers.
As regards to RDSTCs, in the recent literature we can account for some significant works where their performance is analyzed. In [6] and [7] approximated expressions for the outage probability are derived for uniform phase RDSTC. In [8], some of the authors of the current work began to explore the performance of relay networks employing RDSTC in the presence of Rayleigh fading: such an investigation was further extended in [9] to the case of Nakagami fading. The current work generalizes the previous findings, considering the simultaneous presence of Nakagami distributed fast fading and lognormal shadowing. Tight, analytical approximations are derived for both the end-to-end symbol error probability, $S E P_{e 2 e}$, and the end-to-end outage probability, $O P_{e 2 e}$. The approximations are successfully validated through a comparison with the results obtained by simulation, and for the high signal-to-noise ratio (SNR) regime, a much simpler expression is obtained for the $S E P_{e 2 e}$.

The remainder of the paper is organized as follows: Section II provides the channel model description and highlights the notable features of the wireless relay network under consideration; Section III details the analysis that leads to the determination of the $S E P_{e 2 e}$ and of the $O P_{e 2 e}$; Section IV derives an approximated expression for the $S E P_{e 2 e}$, that holds in the asymptotic, high SNR regime; Section V reports some illustrative numerical results and Section VI concludes the paper.

\section{Channel Model And System Description}

The system we consider in this paper is a multiple relay wireless network constituted by a source and a destination terminal that communicate through an array of $Q$ relaying nodes, $R_{1}, R_{2}, \cdots, R_{Q}$. Each node is equipped with a single antenna and is allowed to access the medium in half-duplex mode. Communication relies on a two-hop transmission: on the first hop the source broadcasts the desired message to the array of relay nodes; on the second hop, the relays that are able to correctly decode the message re-encode it through the RDSTC scheme proposed in [2] and forward it to the destination. Synchronization among nodes is maintained in a distributed manner, but detailing its structure is beyond the purpose of this research. 
In what follows, we first put forth the description of the channel model we consider; next, we briefly recall the salient features of the examined relay network.

\section{A. Channel Characterization}

The radio channel between the source and the $j$-th relay is subject to both small and large scale fading phenomena: let $h_{1 j}$ denote its channel coefficient. Similarly, let $h_{2 j}$ denote the coefficient of the radio channel between the $j$-th relay and the destination, that is affected by the same kind of fading. The random variables $h_{i j}, i=1,2, j=1,2, \ldots, Q$, are assumed to be independent; for clearness' sake, in what follows we will omit subscript $i$, discriminating between the two hops, unless strictly necessary. We further assume that $h_{j}$ is Nakagami- $m$ distributed once its mean is fixed, so that the channel gain $g_{j}=\left|h_{j}\right|^{2}$ is Gamma distributed. Accordingly, the probability density function (PDF) of $g_{j}$, conditioned to the channel mean power $\Omega_{j}$, is

$$
p_{\left.g_{j}\right|_{\Omega_{j}}}(x)=\frac{m_{j}^{m_{j}} x^{m_{j}-1}}{\Gamma\left(m_{j}\right) \Omega^{m_{j}}} \exp \left(\frac{-x \cdot m_{j}}{\Omega_{j}}\right),
$$

where $\Gamma(\cdot)$ is the Gamma function, defined as $\Gamma(n+1)=$ $n$ !, and $m_{j}$ is the fading severity parameter; the cumulative distribution function (CDF) of $g_{j}$ conditioned upon $\Omega_{j}$ is

$$
F_{\left.g_{j}\right|_{\Omega_{j}}}(x)=1-\frac{\Gamma\left(m_{j}, \frac{m_{j} x}{\Omega_{j}}\right)}{\Gamma\left(m_{j}\right)},
$$

with $\Gamma(a, x)$ representing the incomplete Gamma function, $\Gamma(a, x)=\int_{x}^{\infty} e^{-t} t^{a-1} d t$. In the literature, $\Omega_{j}$ variations have been described by several distributions: as demonstrated in [10], the one guaranteeing the best agreement with empiric measurements is the lognormal distribution. Hence, in order to account for the effects of shadowing, we model each $\Omega_{j}$ as an independent, lognormally distributed random variable, whose PDF is

$$
p_{\Omega_{j}}(x)=\frac{k}{\sqrt{2 \pi} \sigma_{j} x} \exp \left[\frac{-\left(10 \log _{10} x-\mu_{j}\right)^{2}}{2 \sigma_{j}^{2}}\right],
$$

where $k=10 / \ln 10, \sigma_{j}$ and $\mu_{j}$ being expressed in $\mathrm{dB}$.

Given symbol $\mathbb{E}_{X}[\cdot]$ denotes the expectation with respect to the random variable $X$, to determine the CDF of $g_{j}$ unconditioned to $\Omega_{j}$, we evaluate

$$
F_{g_{j}}(x)=\mathbb{E}_{\Omega_{j}}\left[F_{g_{j} \mid \Omega_{j}}(x)\right] .
$$

Making use of (2) and (3), we can rewrite (4) as

$$
\begin{array}{r}
F_{g_{j}}(x)=\int_{0}^{\infty}\left(1-\frac{\Gamma\left(m_{j}, \frac{m_{j} x}{\Omega_{j}}\right)}{\Gamma\left(m_{j}\right)}\right) \\
\times \frac{k}{\sqrt{2 \pi} \sigma_{j} \Omega_{j}} \exp \left[\frac{-\left(10 \log _{10} \Omega_{j}-\mu_{j}\right)^{2}}{2 \sigma_{j}^{2}}\right] d \Omega_{j},
\end{array}
$$

that with the change of variable $y=\frac{10 \log _{10} \Omega_{j}-\mu_{j}}{\sqrt{2} \sigma_{j}}$ becomes

$$
F_{g_{j}}(x)=\int_{0}^{\infty}\left(1-\frac{\Gamma\left(m_{j}, \frac{m_{j} x}{\beta_{j}(y)}\right)}{\Gamma\left(m_{j}\right)}\right) \frac{\exp \left(-y^{2}\right)}{\sqrt{\pi}} d y,
$$

where

$$
\beta_{j}(y)=10^{\left(\sqrt{2} \sigma_{j} y+\mu_{j}\right) / 10} .
$$

Although a closed-form solution of (6) is not available, a highly accurate discrete approximation is possible resorting to the Gauss-Hermite quadrature method [11], that allows to rewrite (6) as

$$
F_{g_{j}}(x)=1-\sum_{\nu=0}^{N_{H}} \frac{W_{\nu}}{\sqrt{\pi}} \frac{\Gamma\left(m_{j}, \frac{m_{j} x}{\beta\left(r_{\nu}\right)}\right)}{\Gamma\left(m_{j}\right)},
$$

where $W_{\nu}$ and $r_{\nu}$ stand for the $\nu$-th weight and the $\nu$-th root of the Hermite polynomial, respectively, whose values have been tabulated in literature for $N_{H}$ values up to 20 [11]. In the following sections we will employ the approximated expression for the CDF of the channel gain $g_{j}$ provided by (8) to determine both the $S E P_{e 2 e}$ and the $O P_{e 2 e}$ of the transmission system under consideration.

\section{B. System Model}

In the examined scenario, where the radio channel introduces deep fades and the line of sight between any transmitterreceiver pair is seldom present, it is very likely that not all the relays will succeed in decoding the source message. Both the number $T$ and the subset of successful relaying nodes, that we term active nodes, will therefore be random: as indicated by [2], this makes the adoption of RDSTCs particularly attractive. The structure of the complex Gaussian RDSTC considered in this paper is the one first introduced in [2], that is briefly reported below.

Given a block of symbols $\mathbf{s}=\left\{s_{1}, \ldots, s_{B}\right\}$ is broadcasted by the source on the first hop and correctly decoded by $T$ out of the $Q$ relays, then each of the active relays maps the symbols into a matrix $\mathbf{C}$, of size $B \times L$, where $L$ is the number of antennas of the underlying space-time coding scheme; next, every relay provides to retransmit a random linear combination of the encoded symbols, in a manner that is independent from relay to relay. In summary:

$$
\mathbf{s} \rightarrow \mathbf{C}(\mathbf{s}) \rightarrow \mathbf{C}(\mathbf{s}) \mathbf{R},
$$

with $\mathbf{R}$ being the $L \times T$ randomization matrix: its $j$-th column, $\mathbf{r}_{j}$, gives the coefficients of the linear combination performed by the $j$-th relay. The elements of $\mathbf{R}$ are complex Gaussian random variables with zero mean and unit variance.

At the destination, the block of received symbols is

$$
\mathbf{y}=\mathbf{C}(\mathbf{s}) \mathbf{R h}_{2}+\mathbf{n},
$$

where $\mathbf{h}_{\mathbf{2}}=\left[h_{2 j}\right]$ is the $T \times 1$ vector of channel coefficients on the second hop and $\mathbf{n}$ is the vector representing the additive white Gaussian noise.

\section{END-TO-END ANALYSIS}

\section{A. First Hop}

In the previous section, it has been pointed out that due to the fading and shadowing impairments, the set of active relays on the second hop will be a random subset of the whole set of $Q$ relays of the system. Recalling [8], we can therefore express 
the probability of a symbol error or of an outage event $X$ as follows

$$
\operatorname{Pr}(X)=\sum_{z=0}^{Q} \sum_{\mathcal{D}_{z}} \operatorname{Pr}\left(X \mid \mathcal{D}_{z}\right) \operatorname{Pr}\left(\mathcal{D}_{z}\right)
$$

where $\mathcal{D}_{z}$ is a given subset of $z$ active relays. Given that the $j$-th relay is active if it can successfully decode the symbols it receives from the source, i.e., if $\frac{1}{2} \log _{2}\left(1+g_{1 j} \gamma_{0}\right) \geq R_{1}$, where $\gamma_{0}$ is the average SNR and $R_{1}$ the spectral efficiency on the first hop, we have

$$
\begin{gathered}
\operatorname{Pr}\left(D_{z}\right)=\prod_{j \in D_{z}} \operatorname{Pr}\left(g_{1 j}>\phi_{1}\right) \cdot \prod_{j \notin D_{z}} \operatorname{Pr}\left(g_{1 j} \leq \phi_{1}\right), \\
\text { with } \phi_{1}=\frac{2^{2 R_{1}}-1}{\gamma_{0}},
\end{gathered}
$$

that recalling $(8)$ becomes

$$
\begin{aligned}
& \operatorname{Pr}\left(\mathcal{D}_{z}\right)=\prod_{j \in D_{z}} \sum_{\nu=0}^{N_{H}} \frac{W_{\nu}}{\sqrt{\pi}} \frac{\Gamma\left(m_{1 j}, \frac{m_{1 j} \phi_{1}}{\beta_{1 j}\left(r_{\nu}\right)}\right)}{\Gamma\left(m_{1 j}\right)} \\
& \times \prod_{j \notin D_{z}}\left(1-\sum_{\nu=0}^{N_{H}} \frac{W_{\nu}}{\sqrt{\pi}} \frac{\Gamma\left(m_{1 j}, \frac{m_{1 j} \phi_{1}}{\beta_{1 j}\left(r_{\nu}\right)}\right)}{\Gamma\left(m_{1 j}\right)}\right) .
\end{aligned}
$$

\section{B. Symbol Error Probability}

In this subsection, we first derive the analytical expression of $P_{e 2}$, the symbol error probability for the transmission on the second hop of the RDSTC relay network, when an $M$-PSK modulation scheme is considered, resorting to a moment generating function (MGF) based approach. Next, $P_{e 2}$ expression is employed to determine the $S E P_{e 2 e}$. To evaluate $P_{e 2}$, we have to analyze the statistical behavior of $\xi$, the instantaneous received SNR. If we assume as in [8] an orthogonal design of the space-time code, then $\xi$ is given by

$$
\xi=\xi_{0}\left\|\mathbf{R} \mathbf{h}_{\mathbf{2}}\right\|_{F}^{2},
$$

with $\xi_{0}=P_{S} / N_{0}$ being the average SNR at the destination and $\|\cdot\|_{F}^{2}$ indicating the squared Frobenius vectorial norm. Recalling [8], the expression of the MGF of $\xi$ is

$$
\phi_{\xi}(s)=\mathbb{E}_{\left\|\mathbf{h}_{\mathbf{2}}\right\|_{F}^{2}}\left[\left(1+s \xi_{0}\left\|\mathbf{h}_{\mathbf{2}}\right\|_{F}^{2}\right)^{-L}\right] .
$$

As the elements of vector $\mathbf{h}_{\mathbf{2}}$ are independent random variables, (15) can be rewritten via a product form:

$$
\phi_{\xi}(s)=\prod_{j=1}^{T} \phi_{\xi_{j}}(s)
$$

where

$$
\phi_{\xi_{j}}(s)=\mathbb{E}_{\Omega_{2 j}}\left[\mathbb{E}_{g_{2 j} \mid \Omega_{2 j}}\left(1+s \xi_{0} g_{2 j}\right)^{-L}\right] .
$$

Recalling (1) and (3), (17) becomes

$$
\begin{array}{r}
\phi_{\xi_{j}}(s)=\int_{0}^{\infty} \int_{0}^{\infty}\left(1+s \xi_{0} x\right)^{-L} \cdot \frac{m_{2 j}^{m_{2 j}} x^{m_{2 j}-1}}{\Gamma\left(m_{2 j}\right) \Omega_{2 j}^{m_{2 j}}} \\
\times \exp \left(-\frac{x m_{2 j}}{\Omega_{2 j}}\right) \cdot \frac{k}{\sqrt{2 \pi} \sigma_{2 j} \Omega_{2 j}} \\
\times \exp \left[-\frac{\left(10 \log _{10} \Omega_{2 j}-\mu_{2 j}\right)^{2}}{2 \sigma_{2 j}^{2}}\right] d x d \Omega_{2 j} .
\end{array}
$$

Integrating first with respect to $x$ and introducing a suitable change of variable, $q=s \xi_{0} x$, we have

$$
\begin{aligned}
\phi_{\xi_{j}}(s)=\int_{0}^{\infty} & \int_{0}^{\infty}(1+q)^{-L} \cdot \frac{\left(\frac{m_{2 j}}{s \xi_{0}}\right)^{m_{2 j}} q^{m_{2 j}-1}}{\Gamma\left(m_{2 j}\right) \Omega_{2 j}^{m_{2 j}}} \\
& \times \exp \left(-\frac{q m_{2 j}}{s \xi_{0} \Omega_{2 j}}\right) d q \\
\times \frac{k}{\sqrt{2 \pi} \sigma_{2 j} \Omega_{2 j}} & \cdot \exp \left(-\frac{\left(10 \log _{10} \Omega_{2 j}-\mu_{2 j}\right)^{2}}{2 \sigma_{2 j}^{2}}\right) d \Omega_{2 j} .
\end{aligned}
$$

The inner integral can be expressed via the Confluent Hypergeometric function, defined as $\Psi(\alpha, \gamma ; z)=\frac{1}{\Gamma(\alpha)}$. $\int_{0}^{\infty} e^{-z q} q^{\alpha-1}(1+q)^{\gamma-\alpha-1} d q[12$, eq. 9.211-4], so that (19) becomes

$$
\begin{gathered}
\phi_{\xi_{j}}(s)=\int_{0}^{\infty} z_{j}^{\alpha_{j}} \Psi\left(\alpha_{j}, \gamma_{j} ; z_{j}\right) \\
\times \frac{k}{\sqrt{2 \pi} \sigma_{2 j} \Omega_{2 j}} \exp \left(-\frac{\left(10 \log _{10} \Omega_{2 j}-\mu_{2 j}\right)^{2}}{2 \sigma_{2 j}^{2}}\right) d \Omega_{2 j},
\end{gathered}
$$

where

$$
\alpha_{j}=m_{2 j}, \gamma_{j}=m_{2 j}-L+1 \text { and } z_{j}=\frac{m_{2 j}}{s \xi_{0} \Omega_{2 j}} .
$$

To perform the second integration with respect to $\Omega_{2 j}$, it is again convenient to introduce $y=\frac{10 \log _{10} \Omega_{2 j}-\mu_{2 j}}{\sqrt{2} \sigma_{2 j}}$ and rewrite (21) as

$$
\phi_{\xi_{j}}(s)=\int_{0}^{\infty}\left(z_{j}^{\prime}(y)\right)^{\alpha_{j}} \Psi\left(\alpha_{j}, \gamma_{j} ; z_{j}^{\prime}\right) \cdot \frac{\exp \left(-y^{2}\right)}{\sqrt{\pi}} d y
$$

where

$$
z_{j}^{\prime}(y)=\frac{m_{2 j}}{s \xi_{0} \beta_{j}(y)},
$$

and $\beta_{j}(y)$ is defined as in (7) (with $\sigma_{2 j}$ and $\mu_{2 j}$ in place of $\sigma_{j}$ and $\mu_{j}$ ). The integral in (23) is analogous to the one in (6), we therefore resort to the Gauss-Hermite quadrature method and express $\phi_{\xi_{j}}(s)$ as

$$
\phi_{\xi_{j}}(s)=\sum_{\nu=1}^{N_{H}} \frac{W_{\nu}}{\sqrt{\pi}} \cdot\left[z_{j}^{\prime}\left(r_{\nu}\right)\right]^{\alpha_{j}} \Psi\left(\alpha_{j}, \gamma_{j}, z_{j}^{\prime}\left(r_{\nu}\right)\right)
$$

Replacing (25) in (16), the MGF of the instantaneous received SNR at the destination turns out to be

$$
\phi_{\xi}(s)=\prod_{j=1}^{T} \sum_{\nu=1}^{N_{H}} \frac{W_{\nu}}{\sqrt{\pi}}\left[z_{j}^{\prime}\left(r_{\nu}\right)\right]^{\alpha_{j}} \Psi\left(\alpha_{j}, \gamma_{j}, z_{j}^{\prime}\left(r_{\nu}\right)\right) .
$$

We now recall the analysis conducted in [10] and determine $P_{e 2}$ integrating (26) as follows:

$$
P_{e 2}=\int_{0}^{\pi-\frac{\pi}{M}} \frac{1}{\pi} \cdot \phi_{\xi}\left(\frac{\gamma}{\sin ^{2} \lambda}\right) d \lambda
$$

where $\gamma=\sin (\pi M)^{2}$ is the modulation constant. Finally, from (11), (13), (26) and (27), the expression for the $S E P_{e 2 e}$ 
of RDSTC over independent, non identically distributed composite wireless channels turns out to be

$$
\begin{gathered}
S E P_{e 2 e}=\prod_{i \in D_{z}} \sum_{\nu=0}^{N_{H}} \frac{W_{\nu}}{\sqrt{\pi}} \frac{\Gamma\left(m_{i 1}, \frac{m_{i 1} \phi_{1}}{\beta(\nu)}\right)}{\Gamma\left(m_{i 1}\right)} \\
\times \prod_{i \notin D_{z}}\left[1-\sum_{\nu=0}^{N_{H}} \frac{W_{\nu}}{\sqrt{\pi}} \frac{\Gamma\left(m_{i 1}, \frac{m_{i 1} \phi_{1}}{\beta(\nu)}\right)}{\Gamma\left(m_{i 1}\right)}\right] \\
\times \int_{0}^{\pi-\frac{\pi}{M}} \frac{1}{\pi} \prod_{j=1}^{T} \sum_{\nu=1}^{N_{H}} \frac{W_{\nu}}{\sqrt{\pi}}\left[\left.z_{j}^{\prime}\left(r_{\nu}\right)\right|_{s=\gamma / \sin ^{2} \lambda}\right]^{\alpha_{j}} \\
\times \Psi\left(\alpha_{j}, \gamma_{j},\left.z_{j}^{\prime}\left(r_{\nu}\right)\right|_{s=\gamma / \sin ^{2} \lambda}\right) d \lambda .
\end{gathered}
$$

Equation (28) can be readily implemented with the help of standard library functions, available in packages such as MAPLE and MATHEMATICA, with limited resource involvement.

\section{Outage Probability}

The outage is defined as the event where $\xi$, the instantaneous received SNR on the second hop, drops below a given threshold level $\xi_{\text {thres }}$, so that the outage probability $O P$ is

$$
O P=\operatorname{Pr}\left\{\xi \leq \xi_{\text {thres }}\right\} .
$$

Its evaluation requires the $\mathrm{CDF} F_{\xi}(\cdot)$ of the instantaneous received $\mathrm{SNR} \xi$. To obtain such $\mathrm{CDF}$, we recall that

$$
F_{\xi}(\cdot)=\mathcal{L}^{-1}\left(\frac{\phi_{\xi}(s)}{s}\right),
$$

where $\mathcal{L}^{-1}(s)$ denotes the inverse Laplace transform. Following the approach undertaken in [8], we provide an approximated expression for it, that replaced in (11) allows to write the $O P_{e 2 e}$ as

$$
\begin{gathered}
O P_{e 2 e}=\prod_{i \in D_{z}} \sum_{\nu=0}^{N_{H}} \frac{W_{\nu}}{\sqrt{\pi}} \frac{\Gamma\left(m_{i 1}, \frac{m_{i 1} \phi_{1}}{\beta(\nu)}\right)}{\Gamma\left(m_{i 1}\right)} \\
\times \prod_{i \notin D_{z}}\left[1-\sum_{\nu=0}^{N_{H}} \frac{W_{\nu}}{\sqrt{\pi}} \frac{\Gamma\left(m_{i 1}, \frac{m_{i 1} \phi_{1}}{\beta(\nu)}\right)}{\Gamma\left(m_{i 1}\right)}\right] \\
\times\left[\frac{e^{\frac{A}{2}}}{2^{Q} \xi_{t r}} \sum_{g=0}^{G}\left(\begin{array}{c}
G \\
g
\end{array}\right) \sum_{p=0}^{P+g} \frac{(-1)^{p}}{\beta_{p}} \Re\left\{\frac{\phi_{\gamma}\left(\frac{A+2 \pi j p}{2 \xi_{t r}}\right)}{\frac{A+2 \pi j p}{2 \xi_{t r}}}\right\}+o(\xi)\right]
\end{gathered}
$$

where $A, Q$ and $P$ are predefined integers, $\beta_{p}=2$ if $p=0$ and $\beta_{p}=1$ if $p=1,2, \ldots, P, \Re\{\cdot\}$ indicates the real part of its argument and $j^{2}=-1$.

In Section V, we will demonstrate by direct comparison against simulative results that (28) and (31) provide excellent approximations of the exact $S E P_{e 2 e}$ and $O P_{e 2 e}$, respectively.

\section{Asymptotic ANALYSis FOR High SNR}

This section presents the asymptotic analysis of the RDSTC relay system in the high SNR regime and puts forth a far simpler and tight approximation for the $S E P_{e 2 e}$.

To begin with, we observe that in high SNR conditions $\xi_{0} \gg$ 1: in (17) we therefore approximate $1+s \xi_{0} g_{2 j} \simeq s \xi_{0} g_{2 j}$ and write (16) as follows:

$$
\phi_{\xi}(s)=\prod_{j=1}^{T} \mathbb{E}_{\Omega_{2 j}}\left[\mathbb{E}_{g_{2 j}}\left(s \xi_{0} g_{2 j}\right)^{-L}\right] .
$$

In order to evaluate (32), we have to solve the integral

$$
\begin{aligned}
\phi_{\xi_{j}}(s)=\int_{0}^{\infty} & \int_{0}^{\infty}\left(s \xi_{0} x\right)^{-L} \cdot \frac{m_{2 j}^{m_{2 j}} x^{m_{2 j}-1}}{\Gamma\left(m_{2 j}\right) \Omega_{2 j}^{m}} \cdot \exp \left(\frac{-x m_{2 j}}{\Omega_{2 j}}\right) \\
& \times \frac{k}{\sqrt{2 \pi} \sigma_{2 j} \Omega_{2 j}} \\
& \times \exp \left(\frac{-\left(10 \log _{10} \Omega_{2 j}-\mu_{2 j}\right)^{2}}{2 \sigma_{2 j}^{2}}\right) d x d \Omega_{2 j} .
\end{aligned}
$$

Recalling [12, eq. 3.351-4] and applying the Gauss-Hermite quadrature method we write

$$
\begin{aligned}
\phi_{\xi_{j}}(s) & =\sum_{\nu=1}^{N_{H}} K_{\nu} \frac{m_{2 j}^{m_{2 j}}}{\Gamma\left(m_{2 j}\right) \beta\left(r_{\nu}\right)^{m_{2 j}}\left(s \xi_{0}\right)^{L}} \\
\times & \frac{m_{2 j}^{-\left(m_{2 j}-L-1\right)-1}\left(m_{2 j}-L-1\right) !}{\beta\left(r_{\nu}\right)^{-\left(m_{2 j}-L-1\right)-1}},
\end{aligned}
$$

with $K_{\nu}=\frac{W_{\nu}}{\sqrt{\pi}}$, that can be further simplified into

$$
\phi_{\xi_{j}}(s)=\sum_{\nu=1}^{N_{H}} K_{\nu} \frac{\Gamma\left(m_{2 j}-L\right)\left(s \xi_{0}\right)^{-L}}{\Gamma\left(m_{2 j}\right)}\left(\frac{m_{2 j}}{\beta\left(r_{\nu}\right)}\right)^{L} .
$$

If we introduce the constant

$$
C_{j \nu}=K_{\nu}\left(\frac{m_{2 j}}{\beta\left(r_{\nu}\right)}\right)^{L} \prod_{r=1}^{L} \frac{1}{m_{2 j}-r}
$$

we can rewrite $\phi_{\xi_{j}}(s)$ as

$$
\phi_{\xi_{j}}(s)=C_{j \nu} \cdot\left(s \xi_{0}\right)^{-L} .
$$

We next observe that for high SNR values the probability each relay node being active is close to 1 , and therefore assume that in such conditions, we can count on the whole array of relays $R_{1}, R_{2}, \cdots, R_{Q}$. Accordingly, (32) turns into

$$
\phi_{\xi}(s)=\prod_{j=1}^{Q} C_{j \nu} \cdot\left(s \xi_{0}\right)^{-L}
$$

that introducing symbol $B, B=\prod_{j=1}^{Q} C_{j \nu}$, becomes

$$
\phi_{\xi}(s)=B \cdot\left(s \xi_{0}\right)^{-K L} .
$$

It is now straightforward to determine $P_{e 2}$ expression in the high SNR regime: recalling (27) and (39) it is

$$
P_{e 2}=\int_{0}^{\pi-\frac{\pi}{M}} \frac{1}{\pi} B \cdot\left(\frac{\gamma}{\sin ^{2} \lambda} \xi_{0}\right)^{-K L} d \lambda .
$$

In the next section, we will show that adopting (40) to evaluate the $S E P_{e 2 e}$ guarantees a remarkably tight approximation when high SNR values are considered.

\section{Numerical RESUlts}

We report in this section the results obtained for the previously modeled RDSTC relay network when two settings are examined, with different diversity degrees: System 1, where $K=3$ relays are considered and the number of antennas of the underlying space-time code is $L=3$, and System 2, where $K=2$ and $L=2$. The channel parameter values for 


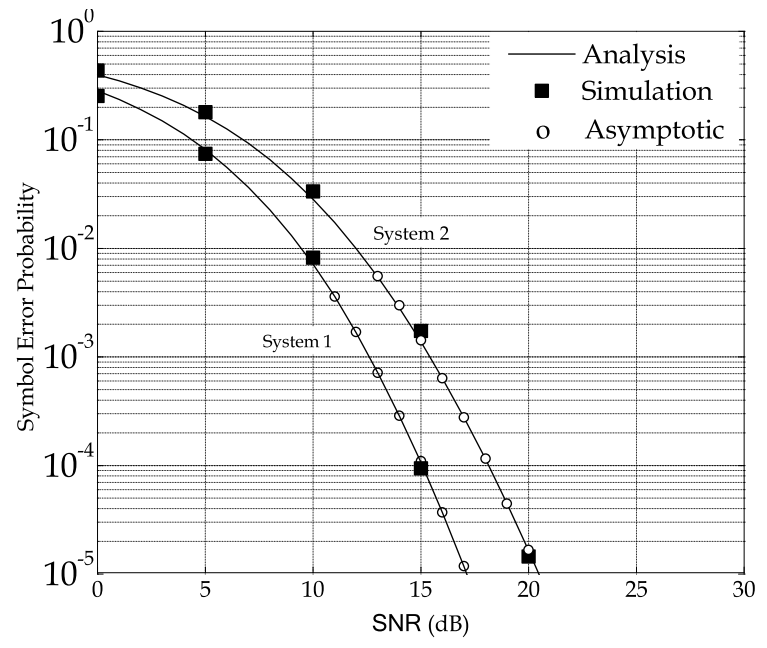

Fig. 1. $S E P_{e 2 e}$ of Complex Gaussian RDSTCs over composite channels.

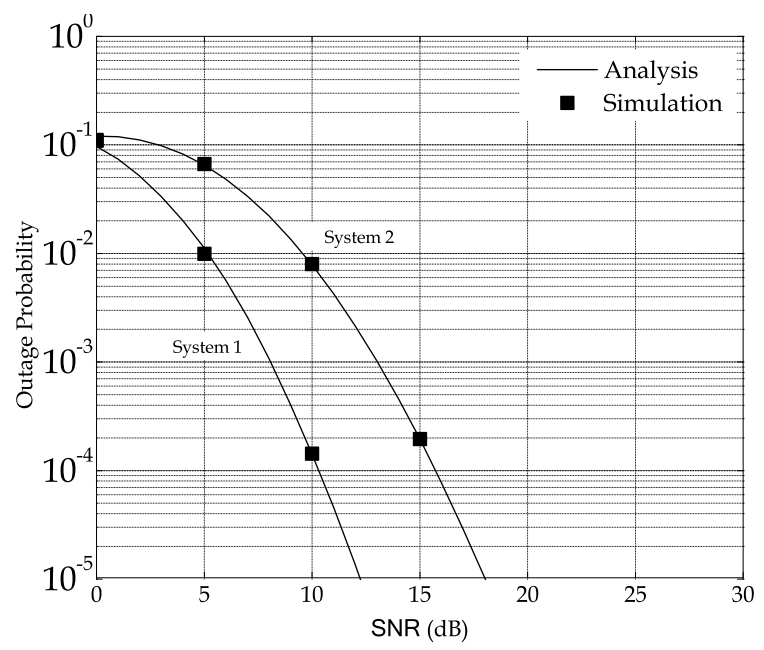

Fig. 2. $O P_{e 2 e}$ of Complex Gaussian RDSTCs over composite channels.

the Nakagami and the lognormal distributions are reported in Table I. The same space-time codes investigated in [13] and [2] are adopted; also, on the first hop we have considered a unitary spectral efficiency, $R_{1}=1 \mathrm{bit} / \mathrm{s} / \mathrm{Hz}$, and the modulation scheme on the second hop is 8-PSK. In order to determine our results we have performed the Gauss-Hermite quadrature integration with a Hermite polynomial of degree $N_{H}=10$ : the graphs will demonstrate that this choice guarantees an excellent accuracy. Fig. 1 reports the $S E P_{e 2 e}$ behavior as

TABLE I

NAKAGAMI AND LOGNORMAL SHADOWING PARAMETERS

\begin{tabular}{|c|c|}
\hline$\left\{m_{11}, m_{12}, m_{13}\right\}$ & $\{2,1,3\}$ \\
$\left\{m_{21}, m_{22}, m_{23}\right\}$ & $\{4,4,5\}$ \\
\hline$\left\{\sigma_{11}, \sigma_{12}, \sigma_{13}\right\} \mathrm{dB}$ & $\{6,7,8\}$ \\
$\left\{\sigma_{21}, \sigma_{22}, \sigma_{23}\right\} \mathrm{dB}$ & $\{5,6,6\}$ \\
$\left\{\mu_{11}, \mu_{12}, \mu_{13}\right\} \mathrm{dB}$ & $\{-10,-8,-7\}$ \\
$\left\{\mu_{21}, \mu_{22}, \mu_{23}\right\} \mathrm{dB}$ & $\{-8,-10,-7\}$ \\
\hline
\end{tabular}

a function of $\xi$, the $S N R$ expressed in dB: as expected, System 1 outperforms System 2, given the former exhibits a larger diversity gain. It is evident from this figure that there is perfect agreement between the analysis and Monte Carlo simulations. Fig. 1 also indicates that the $S E P_{e 2 e}$ approximation for the high SNR regime is hardly distinguishable from the analytical curve and therefore represents an effective alternative to the exact evaluation. Fig. 2 shows the $O P_{e 2 e}$, and here too, an accurate match between the analytical results and the simulative outcomes is evident.

\section{CONCLUSIONS}

This work has assessed the end-to-end performance of a wireless relay network that relies upon RDSTC with complex Gaussian distribution, when both small and large scale fading phenomena are considered. Extremely tight, analytical approximations of the $S E P_{e 2 e}$ and the $O P_{e 2 e}$ have been derived through the Gauss-Hermite quadrature method. For the high SNR regime, a much simpler, yet accurate expression for the $S E P_{e 2 e}$ has been obtained. The analysis has been successfully validated through Monte-Carlo simulation for selected numerical examples.

\section{REFERENCES}

[1] Y. Jing and B. Hassibi, "Distributed space-time coding in wireless relay networks," IEEE Trans. Wireless Commun., vol. 5, no. 12, pp. 35243536, Dec. 2006.

[2] B. Sirkeci-Mergen and A. Scaglione, "Randomized space-time coding for distributed cooperative communication," IEEE Trans. Signal Process., vol. 55, no. 10, pp. 5003-5017, Oct. 2007.

[3] X. Mestre and D. Gregoratti, "Diversity analysis of randomized linear dispersion codes in a half-duplex amplify-and-forward multiple-relay system," IEEE Trans. Inf. Theory, vol. 59, no. 5, pp. 2936-2959, May 2013.

[4] T. K. C. Nie, P. Liu and E. Erkip, "Cooperative relaying in nextgeneration mobilewimax networks," IEEE Trans. Veh. Technol., vol. 63, no. 3, pp. 1399-1405, Mar. 2013.

[5] N. Suraweera and N. Beaulieu, "Performance analysis of decode-andforward relaying with optimum combining in the presence of co-channel interference," in Proc. IEEE ICC, Budapest, Hungary, Jun. 2013, pp. $4968-4972$.

[6] L. Zhang and L. J. Cimini, "Outage analysis and optimization for discrete randomized distributed space-time block coding," in Proc. IEEE GLOBECOM, Washington DC, U.S.A., Nov. 2007, pp. 4349-4353.

[7] D. Gregoratti and X. Mestre, "Diversity analysis of a randomized distributed space-time coding in an amplify and forward relay channel," in Proc. IEEE ICC, Dresden, Germany, Jun. 2009, pp. 1-5.

[8] E. E. T. Q. Duong, O. Alay and H.-J. Zepernick, "End-to-end performance of randomized distributed space-time codes," in Proc. IEEE PIMRC, Istanbul, Turkey, Sep. 2010, pp. 988-993.

[9] H.-J. Z. J. Soffritti and M. L. Merani, "Performance analysis of randomized distributed space-time codes in Nakagami- $m$ fading," in Proc. IEEE ATC, Ho Chi Minh City, Vietnam, Oct. 2013, pp. 408-413.

[10] M. K. Simon and M.-S. Alouini, Digital Communication over Fading Channels: A Unified Approach to Performance Analysis. New York: Wiley, 2000.

[11] R. Greenwood and J. Miller, "Zeros of the hermite polynomial and weights of the Gauss' mechanical quadrature formula," Bull. Amer. Math. Soc., vol. 54, no. 8, pp. 765-769, 1948.

[12] I. S. Gradshteyn and I. M. Ryzhik, Table of Integrals, Series, and Products, 7th ed. San Diego, CA: Academic, 2007.

[13] O. Tirkkonen and A. Hottinen, "Square-matrix embeddable space-time block codes for complex signal constellations," IEEE Trans. Inf. Theory, vol. 48, no. 2, pp. 384-395, Feb. 2002. 